\title{
Eine Generation im Bildersturm Über das Mediennutzungsverhalten der Jugend
}

\author{
Bernhard Heinzlmaier
}

\section{Zusammenfassung}

Die Jugendwertestudie 2019 und die Studie „Generation Corona“ des Instituts für Jugendkulturforschung beschäftigen sich mit der Frage, welche Ansprüche Jugendliche insbesondere in Krisenzeiten an Medien stellen. Die Ergebnisse legen ein Wiedererstarken von Nationalismus, die Aufwertung von Institutionen und den Staat als Garant für Sicherheit und Stabilität nahe. Dabei spielt die Familie eine wichtige Rolle und das Individuum wird am ästhetischen Aufmerksamkeitskapital gemessen. Für ein erfolgreiches Jugendmedium sind also eine österreichische Ausrichtung sowie Durchsetzungsfähigkeit wichtig. Das Medium sollte Strategien zur Stabilitätsfindung und Gemeinschaftsthemen thematisieren, ohne den Staat oder den Markt anzubiedern. Ästhetisch ist die Anschlussfähigkeit an eine soziokulturelle Mitte wichtig. Das bedeutet auch, dass Bilder eine entscheidende Rolle spielen.

\section{Abstract}

The Youth Value Study 2019 and the "Generation Corona" study by the Institute for Youth Culture Research deal with the question of what demands young people make on media, especially in times of crisis. The results suggest a resurgence of nationalism, the upgrading of institutions and the state as guarantor of security and stability. The family plays an important role here and the individual is measured by the aesthetic attention capital. For a successful youth medium, therefore, an Austrian orientation and assertiveness are important. The medium should focus on strategies for finding stability and community issues without chumming up the state or the market. Aesthetically, the

Katharina Gallner-Holzmann, Theo Hug, Günther Pallaver (Hg.):

Jugendliche Mediennutzung und die Zukunft des Qualitätsjournalismus

(C) 2020 innsbruck university press, ISBN 978-3-99106-014-7, DOI 10.15203/99106-014-7 
ability to connect to a socio-cultural centre is important. This also means that images play a decisive role.

\section{Einleitung}

Der Informationsaustausch findet heute überwiegend vermittelt statt. Zwischen den kommunizierenden Menschen haben sich technische Dispositive etabliert, die Medien. Sie transportieren Botschaften vom Sender zum Empfänger, nicht aber ohne auf deren Wahrnehmung und Wertung massiv Einfluss zu nehmen. Medien sind nicht neutral, im Gegenteil, sie bilden nicht Wirklichkeit ab, sie schaffen Wirklichkeit.

Von Marshall McLuhan stammt der Satz „The Medium is the Massage“. Damit meint McLuhan, dass die Medien förmlich unsere Wahrnehmungsorgane massieren. Die einschmeichelnde Massage, die sie ihren Zielgruppen angedeihen lassen, sollen diese gewogen machen für die Inhalte, die auf das gesamte menschliche Sensorium, zum Beispiel von der kommerziellen und politischen Werbung, losgeschickt werden. Wünsche, Ideologien und Überzeugungen werden sozusagen in die Sinnesorgane „einmassiert“ (Fiore/McLuhan 1967).

Medien standen immer schon unter Manipulationsverdacht. Seit sie aufgrund des technischen Fortschritts zu Massenmedien geworden sind und riesige Reichweiten generieren, werden sie besonders skeptisch betrachtet, nicht nur aufgrund ihrer suggestiven Potentiale, die darin bestehen, Menschen so zu affizieren und emotionalisieren, dass diese am Ende sogar gegen ihre objektiven Interessen handeln, sondern auch wegen der ungleichen Verfügungsgewalt über sie, die in erster Linie beim Staat oder bei finanzstarken Interessensgruppen liegt.

Die technischen Apparaturen, mit denen ein TV-Sender oder Zeitungsverlag betrieben wird, kann sich eben nicht jeder leisten und so sind es wie so oft in der Geschichte die Reichen und Begüterten, die in der Medienwelt das Sagen haben und darüber entscheiden, welche Nachrichten in Umlauf gebracht und wie politische, ökonomische oder kulturelle Sachverhalte dargestellt und interpretiert werden.

Mit der Digitalisierung war lange die Hoffnung verbunden, dass sich das Kräfteverhältnis zwischen mächtigen Sendern und ohnmächtigen Empfängern von Medieninhalten zugunsten der letzteren verschieben könnte. Viele glaubten, mit Hilfe der digitalen Medien würden nun auch dem machtlosen Einzelnen oder kleinen randständigen Interessengemeinschaften die Möglichkeit gegeben, in die Rolle eines einflussreichen Senders 
treten und vom Mainstream abweichende Deutungen des historischen oder Zeitgeschehens in Umlauf bringen zu können.

Aber mitnichten. Die „Demokratisierung“ der medialen Kommunikation stellte sich bald als Illusion heraus, da große internationale Konzerne die Kontrolle über die relevanten Online-Plattformen übernahmen und parallel dazu die traditionellen Print- und elektronischen Medien wie gewohnt in Abhängigkeit von staatlichen oder kommerziellen Eigentümern weiterarbeiteten.

\section{Medienwahrnehmung in Corona-Zeiten}

Gerade in Zeiten der Corona-Krise ist bei vielen jungen Menschen der Eindruck entstanden, dass sich einige der reichweitenstärksten Medien einer staatlichen MessageControl unterworfen haben und bereitwillig dafür sorgen, dass die Maßnahmen der Regierung gegen die Epidemie als ,alternativlos“ erscheinen. Eine offene Diskussion darüber, ob die restriktiven Verordnungen zum Beispiel über die Nutzung des öffentlichen Raumes noch in Einklang mit den verfassungsmäßig garantierten individuellen Freiheitsrechten stehen, wurde so unterbunden.

Doch die Medienkritik der Jugendlichen und jungen Erwachsenen ist keineswegs undifferenziert, vielmehr wird ein deutlicher Unterschied zwischen der Berichterstattung der so genannten Boulevardmedien und den Qualitätsmedien gemacht, egal ob nun der Staat oder privatwirtschaftliche Mächte diese Medien kontrollieren. Kritik wird vor allem an den Boulevardmedien geübt.

Dem Boulevard wird nicht nur vorgeworfen, ein unkritisches Instrument der Bundesregierung gewesen zu sein, sondern auch die Gefährlichkeit des Covid-19-Virus dermaßen übertrieben zu haben, dass unverhältnismäßige Angst und Panik in der Bevölkerung entstand.

Die repräsentative Jugendwertestudie des Instituts für Jugendkulturforschung aus dem April 2020 zeigt, dass über 80\% der unter 30-jährigen den österreichischen Boulevardmedien vorwerfen, die ohnehin vorhandenen Ängste in der Bevölkerung ,,durch reißerische und übertriebene Berichterstattung“" zusätzlich verstärkt zu haben. Demgegenüber werden Qualitätszeitungen wie DER STANDARD und der staatliche ORF als seriöse Informationsquellen wahrgenommen, die sich zumindest bemüht haben, ausgewogen und anständig zu berichten (vgl. Jugendkulturforschung 2020). 
Diese Einschätzung der Medien hat zur Folge, dass sich im April 2020 70\% der österreichischen Jugendlichen mit Hilfe des ORF über die Corona-Epidemie informierten, 32\% über die Tageszeitung DER STANDARD und lediglich 22\% über die auflagenstarke Kronen Zeitung.

Dem österreichischen Boulevard hat die übertriebene und unverhältnismäßige Berichterstattung über die Corona-Epidemie massiv geschadet. Es zeigt sich, dass die Sensationsberichterstattung der Boulevardpresse den überwiegenden Teil der Jugend mehr und mehr skeptisch zurückweichen lässt. Offensichtlich schlägt hier das stark verbesserte Bildungsniveau in den mittleren und unteren Bildungsschichten durch. Selbst die bildungsferneren Milieus lassen sich nicht mehr durch die emotionalisierende und auf Sensationsaspekte verkürzte Wort- und Bildsprache der Regenbogenpresse so leicht beeinflussen und vereinnahmen, wie das noch in den 1980er- und 1990er-Jahren der Fall war. In der Corona-Krise sind die Boulevardmedien jedenfalls nicht die Leitmedien der österreichischen Jugendlichen und jungen Erwachsenen (ebd.).

\section{Die Jugend 2020: Auf der Suche nach geerdeten und glaubwürdigen Medien}

Wie die Untersuchungen des Instituts für Jugendkulturforschung in den letzten Jahren zeigten, ist für Jugendliche bei der Mediennutzung vor allem der Unterhaltungsaspekt relevant. Insbesondere das Internet fungiert dabei als Unterhaltungsabteilung des Medienangebotes (vgl. u.a. Jugendkulturforschung 2019)

Heute sehen wir aber, dass in Krisenzeiten das Unterhaltungsbedürfnis bei der Mediennutzung unter Jugendlichen an Relevanz verliert und demgegenüber verstärkt der Wunsch nach ernsthafter und fundierter Information in den Vordergrund tritt. Das heißt nicht, dass man in der Krise nicht mehr unterhalten werden will, es bedeutet aber, dass der sachlichen und glaubhaften Information eine größere Bedeutung zukommt, vor allem aber dem seriösen Image des Trägers der Berichterstattung überragender Wert beigemessen wird (vgl. Jugendkulturforschung 2020).

Ein Krisenbewusstsein hat sich bei der österreichischen Jugend nicht erst im Zuge der Corona-Krise ausgeprägt. Schon davor empfanden viele die postmoderne Gesellschaft als Abstiegsgesellschaft, in der dem Leistungs- und Wettbewerbsgedanken ein zu großer Einfluss zukommt. Die ständig sich vermehrenden Erfolgskontrollen und Leistungsevaluierungen verunsicherten und stressten die Jugendlichen schon von der Jahrtausend- 
wende weg. Das neue System der permanenten Bewährung wurde immer mehr jungen Menschen einfach zu viel.

Die Zahl derer, die sich den Leistungsanforderungen in Ausbildung und Beruf nicht gewachsen fühlten und deshalb Angst vor dem persönlichen Scheitern und dem daraus folgenden gesellschaftlichen Abstieg haben, ist groß geworden. Viele der Jungen glauben, den Status ihres Elternhauses nicht mehr halten zu können, fürchten sich davor, in eine niedrigere Gesellschaftsschicht abzugleiten.

Wenn alles flüchtig, instabil, unberechenbar, schwankend und unbeständig wird, ist es natürlich, dass der Mensch sich auf die Suche nach Halt begibt. In Österreich sind es bereits um die 70\% der unter-30-jährigen, die unumwunden bekennen, dass sie „Halt im Leben" suchen. Die Frage ist nun, auf welche Ideologien, Institutionen, Dispositive sich diese Suche ausrichtet. Wer oder was wird um Halt und Stabilität angerufen?

Auf der ideologischen Ebene ist es die Nation. Das Österreichbewusstsein, die Loyalität und Liebe zur Heimat feiert eine Renaissance. Im Jahr 2019 zeigten sich 3/4 der Jugendlichen stolz darauf, Österreicher zu sein. Im ländlichen Raum ist dieser Nationalstolz deutlich stärker ausgeprägt als in der Metropole Wien. Aber insgesamt sehen wir, dass der überwiegende Teil der österreichischen Jugend sich am Prinzip des deutschen Philosophen Odo Marquard, „Zukunft braucht Herkunft“ (Marquard 2003) zu orientieren scheint. Der bedrohlichen und unberechenbaren globalisierten Welt wird die vertraute Gemeinschaft der Herkunftsgleichen entgegengesetzt.

Die verunsicherten Jugendlichen, die um Halt und Stabilität bangen, suchen aber auch Schutz und Sicherheit bei starken und etablierten Institutionen. An der Spitze steht dabei die Polizei, gefolgt von den Gerichten, Gewerkschaften und dem Bundesheer. Wenig vertraut man den Parteien und den Managern. Zweitere werden offenbar als die personalen Träger einer Globalisierung, die den Jungen Zuversicht, Sicherheit und berechenbare Verhältnisse raubt, gesehen.

Aber Halt und Sicherheit gewinnt man auch durch die psychische Stärkung des eigenen Selbst, durch die Aufladung des eigenen Ich mit Symbolen der Vitalität, der Wettbewerbstauglichkeit und des individuell erworbenen Erfolges, der erfolgreichen Inszenierung einer ästhetischen Singularität über die Aneignung von attraktiven körperbezogenen und vestimentären Stilelementen.

Letzteres, vor allem aber die symbolische Kompetenz und die ästhetische Selbstinszenierungsfähigkeit sind wichtig in einer performativen Wettbewerbskultur, in der Status 
nicht mehr über die Leistungserbringung, sondern über den Leistungsverkauf erworben wird (Jugendkulturforschung 2019).

Fasst man alle Daten zusammen, die um die Themen der Suche nach Halt und Sicherheit und um die sich daraus ergebenden ideologischen und institutionellen Konsequenzen kreisen, so findet man folgendes komplexes Dispositiv. Es setzt sich zusammen aus

- der wiedererstarkten Ideologie des Nationalismus,

- der Aufwertung von mächtigen Institutionen,

- dem Wiedererstarken des Staates als Garant von Sicherheit/Stabilität,

- dem Bedeutungsgewinn der bürgerlichen Familie,

- dem Aufschwung des Diskurses über Konventionalismus und Normentreue,

- die zustimmende Rezeption des Diskurses zu einem vom Staat kontrollierten Markt und seinen Normen der Konkurrenz und des Wettbewerbs,

- und zuletzt aus der Zustimmung zur Ideologie einer performativen Ökonomie, einer Aufmerksamkeitskultur, die den Wert des Individuums an seiner ästhetischen Ausstattung und des Aufmerksamkeitskapitals bemisst, das er auf den Offline- und Online-Selbstdarstellungsbühnen erwirbt (vgl. Jugendkulturforschung 2019 und Jugendkulturforschung 2020).

Vor dem Hintergrund des oben dargestellten Dispositivs zeigen sich nun deutlich die Konturen des Idealtypus eines zukunftsfähigen Jugendmediums. Dieses muss folgende Eigenschaften beinhalten:

- Es sollte ein österreichisches Medium sein.

- Es muss ein starkes Medium sein, das praktisch und symbolisch seine Durchsetzungsfähigkeit nach- und aufzuweisen versteht.

- Es muss sich Themen der Sicherheit und Stabilität widmen und nützliche Taktiken und Strategien der Sicherheits- und Stabilitätsgewinnung vermitteln, die sich in die Lebenswelt von jungen Menschen integrieren lassen.

- Es muss Jugendliche als Gemeinschafts- und Familienwesen wahrnehmen und auch als solche adressieren.

- Es darf sich weder auf die Seite einer inhumanen Marktideologie, in der der Mensch den Kräften des Marktes schutzlos ausgeliefert ist, noch auf die Seite eines übermächtigen Sicherheitsstaates stellen, der alle Singularitäten und Selbstinitiativen erstickt.

- Es inszeniert sich als ästhetisch kompetentes Medium, dessen ästhetische Konzepte niemals die Anschlussfähigkeit an die konventionalistische und normentreue soziokulturelle Mitte verliert. 


\section{Zum Schluss: Der Megatrend zur präsentativen Symbolik}

Der Schriftsteller und Dandy Oscar Wilde, verstorben im Jahr 1900 in Paris, hat eine seiner literarischen Figuren folgende Feststellung treffen lassen: „In Angelegenheit von großer Wichtigkeit kommt es nicht auf den Ernst, sondern auf den Stil an“" (Wilde 18905 , o.S.). Es gibt wohl kein Zitat, das besser die kommunikative, aber auch die ökonomische und politische Kultur unserer Zeit beschreiben könnte. Wichtig ist nicht das, was man sagt und tut, sondern die Art und Weise, wie man es sagt und wie man es tut. Es dominiert die Form längst den Inhalt. Stil ist gefragt anstelle von Wahrhaftigkeit und Authentizität.

Selten stolpert heute ein Politiker oder ein Wirtschaftsmanager über das, was er getan hat, aber häufig darüber, wie er darüber spricht, wie er über die Sache spricht und vor allem, wie er sich selbst präsentiert.

Ein gutes Beispiel dafür ist Ulrike Lunacek. Sie muss gehen, weil sie nicht den richtigen Stil im Dialog mit den maßgeblichen Kunstmanagern und Künstlern des Landes gefunden hat und weil sie bei einer Pressekonferenz „unbeholfen“ agierte (derStandard 2020).

Über Erfolg und Misserfolg entscheiden Form und Stil einer Pressekonferenz. Und wer noch dazu nicht so aussieht, wie man sich das in Künstlerkreisen vorstellt, den zerreißt das Staatskabarett des ORF. „Und ich als ISTAZ - inkompetenteste Staatssekretärin aller Zeiten - kann euch versichern: Die Kunst ist relativ wichtig“, so der Kabarettist Grissemann in seiner Parodie (Willkommen Österreich 2020). Hätte sie sich besser selbstinszeniert, dann wäre alles gut gewesen. Denn entscheiden kann eine Staatssekretärin ohnehin nichts. Entscheidungen trifft der ihr vorgesetzte Minister. Der hat sich noch keine ironische Verhöhnung eingefangen. Offenbar hat er den richtigen Stil.

Erfolg ist heute eng verbunden mit der Fähigkeit, Bilder zu erzeugen, egal ob es sich um Sprachbilder oder um fotografisches oder filmisches Material handelt. Warum das so ist? Weil die Menschen keine rationalen Wesen, sondern emotionale Wesen sind. Sie entscheiden geleitet von Affekten und Emotionen. Die Vernünftigkeit der Entscheidung konstruieren sie ex post dazu. Man wählt Politiker, weil sie gut aussehen, durchsetzungsstark auftreten und rhetorisch brillant sind und man wählt sie sogar, wenn sie die objektiven Interessen der Wähler mit Füßen treten. Gewählt wird der, mit dem man sich stilistisch identifizieren oder zu dem man aufschauen kann. Gewählt wird nicht, wer so hinfällig und schwach ist wie man selbst und nur über die Defizite jammert, unter denen die Armen leiden. 
Unterm Strich: die durchschnittliche Mehrheit folgt dem schönsten Bild und nicht dem scharfsinnigsten Analytiker, außer er verfügt über die Fähigkeit, Menschen einnehmende Sprachbilder zu erzeugen. Der französische Psychologe Le Bon hat es zur Wende vom 19. zum 20. Jahrhundert so ausgedrückt: „Die Massen können nur in Bildern denken und lassen sich nur durch Bilder beeinflussen. Nur diese schrecken oder verführen sie und werden zu Ursachen ihrer Taten." (Le Bon 1895). Hart und etwas herablassend ausgedrückt, aber wahr, vor allem für unsere Gegenwart.

Warum? Weil wir, die Masse, heute oberflächlicher denn je sind. Wir haben keine Zeit oder wir nehmen sie uns nicht dafür, uns mit den Dingen eingehend und intensiv zu beschäftigen. Texte, Bilder, Meme rauschen in Überfülle an uns vorbei. Wir wollen möglichst viel davon mitbekommen und um anderes, das möglichweise viel interessanter ist als das, was wir gerade im Blickfeld haben, nicht zu versäumen, berühren wir alles nur flüchtig mit der Spitze unserer Augen. Wenn diese Art von Wahrnehmung von irgendetwas angerührt oder berührt wird, dann muss das schon etwas Außergewöhnliches sein. Die Aufmerksamkeit der Menschen, die nach dem Wahrnehmungsmodus der Hyperattention funktioniert, gewinnt der, der die besten und die am schnellsten zu verarbeitenden, die eindeutigsten Stimuli liefert.

Was nun bedeutet der Begriff „Hyperattention“? Wie funktioniert ein Mensch, dessen Aufmerksamkeitssystem in diesem Modus läuft? Hyperattention bedeutet, so formuliert es die amerikanische Wissenschaftlerin Katherine Hayles, das Bedürfnis nach schnellen Wechseln zwischen verschiedenen Aufgaben, simultane Mediennutzung, extrem hohes Stimulationsbedürfnis und geringe Toleranz für Langeweile (Hayles 2007, 187).

Früher funktionierte die Menschheit nach dem Modus der Deep attention. Sie kennzeichnet die Fähigkeit zur langanhaltenden Konzentration auf eine Sache, geringe Ablenkbarkeit durch Stimulation von außen, Nutzung einer einzigen Medienquelle.

Der Bruch zwischen den Generationen verläuft an der Grenze zwischen den skizzierten beiden Aufmerksamkeitskulturen. Wer die Jungen erreichen will, muss sich von den alten Konzepten einer diskursiven Symbolik der Kommunikation, die zu weit ausholenden Erklärungen mit Hilfe des logischen Instruments der Sprache neigt, verabschieden und stärker auf Formen der präsentativen Symbolik setzen (Hayles 2007).

Die präsentative Symbolik der Kommunikation setzt auf den Ausdruck durch Bilder und mimetisch-körperliche Gesten, auf sprachlose Zeichen und auf Musik. Präsentative Symbolik setzt darauf, dass sie sinnlich unmittelbar wirkt und intuitiv verstanden werden kann. 
Für die amerikanische Philosophin Susanne K. Langer ist es vor allem die visuelle Kommunikation, die einer nicht rational-argumentativen, präsentativen und holistischen Logik folgt. Sie unterscheidet sich darin von der argumentierenden Logik der Textkommunikation (Langer 1980).

Will man die jungen Zielgruppen, und wir sprechen hier von den unter 40-Jährigen, erreichen, so kommt man nicht darum herum, Elemente der beschriebenen präsentativen Symbolik stärker in die verwendeten Kommunikationsmittel zu integrieren. Nur so ist es möglich, jene jungen Menschen zu erreichen, deren Gehirne im Modus der Hyperattention arbeiten und das bedeutet, sie wollen den schnellen Wechsel, konsumieren verschiedene Medien simultan und wollen durch außergewöhnliche Reize stimuliert werden.

Abschließend noch ein wichtiger Hinweis: Wer das Bild in der Kommunikation privilegiert, der bedient sich eines irrationalen, affizierenden und emotionalen Mediums, das zu täuschen und zu manipulieren versteht, das nur so tut, als wäre es real. Bei der Verwendung des irrationalen Bildes gilt es, immer die moralischen Grenzen auszuloten, an denen entlang man sich bewegt.

Andererseits hat das Bild aber unbestreitbar einen Vorteil für den Erfolg vieler Kommunikationsmaßnahmen. So wird es besser vom Rezipienten erinnert, man spricht sogar vom sogenannten „Picture Superiority Effect“, das heißt, es erregt stärker Aufmerksamkeit als das geschriebene Wort. Hinzu kommt, dass es stärker zum Handeln motiviert und mit recht geringer kognitiver Kontrolle rezipiert wird, was bedeutet, dass es kaum von moralischen oder ideologischen Barrieren abgeblockt werden kann. Es ist also schwer für den Rezipienten, zum Bild auf kritische Distanz zu gehen.

Man kann nun sagen, die Kommunikation mit präsentativen Symboliken wie Bildern und Musik, mit allem was sinnlich unmittelbar wirkt und intuitiv verstanden wird, ist unmoralisch. Möglicherweise. Aber die Büchse der Pandora ist längst geöffnet und all diese freigelassenen Übel sind nicht mehr rückholbar. So auch die visuelle Kommunikation. Ist man zu einer pragmatischen Handlungsweise fähig, sollte man sich ihrer Vorzüge und Möglichkeiten bedienen, ist man es nicht, bleibt man moralisch sauber im Feld der argumentierenden Logik der Textkommunikation zurück. Man hat dann mit einem zwar erlesenen, wenngleich recht kleinen Publikum zu rechnen. 


\section{Literatur}

derStandard (2020): Sieben Grüne No-Gos. Bericht vom 25.April 2020. Online abrufbar unter: https://www.derstandard.de/story/2000117099328/sieben-gruene-no-gos (abgerufen am 2.6.2020).

Grissemann, Christoph (2020): Willkommen Österreich vom 21.4.2020. Online abrufbar unter: https://www.youtube.com/watch?v=U9 Ccvp67oI (abgerufen am 2.6.2020).

Hayles, Katherine (2007): Hyper and Deep Attention. The Generational Divide in Cognitive Modes. In: Profession (2007) herausgegeben von Modern Language Association, 187-199. Online unter: https://www.jstor.org/stable/25595866?seq=1\#metadata info tab contents (abgerufen am 2.6.2020).

Institut für Jugendkulturforschung (2019): Jugendwertestudie 2019. Soziale Medien, Soziale Bewegungen und Politik. In Auszügen online abrufbar unter: https://jugendkultur.at/jugendwertestudie-2019/ (abgerufen am 2.6.2020).

Institut für Jugendkulturforschung (2020): Generation Corona: die Studie. In Auszügen online abrufbar unter: https://jugendkultur.at/generation-corona-rueckzug-vomrueckzug/ (abgerufen am 2.6.2020).

Langer, Susanne (1980): Philosophie auf neuem Wege. Das Symbol im Denken, im Ritus und in der Kunst. München: Fischer Wissenschaft.

Le Bon, Gustave (1895): Die Psychologie der Massen. Aus dem Französischen von Rudolf Eisler. 2. Auflage Leipzig 1912. Nachdruck Köln 2016.

Mc Luhan, Marshall/ Fiore, Quentin (1967): The Medium is the Massage. London: Penguin Books.

Wilde, Oscar (1895): The Importance of Being Earnest. Online unter: https://www.gutenberg.org/files/844/844-h/844-h.htm (abgerufen am 12.6.2020). 